\title{
Population Balance Model of Heat Transfer in Gas-Solid Processing Systems
}

\author{
Béla G. Lakatos \\ University of Pannonia, Veszprém \\ Hungary
}

\section{Introduction}

In modeling heat transfer in gas-solid processing systems, five interphase thermal processes are to be considered: gas-particle, gas-wall, particle-particle, particle-wall and wall-environment. In systems with intensive motion of particles, the particle-particle and particle-wall heat transfers occur through inter-particle and particle-wall collisions so that both experimental and modeling study of these collision processes is of primary interest.

For modeling and simulation of collisional heat transfer processes in gas-solid systems, an Eulerian-Lagrangian approach, with Lagrangian tracking for the particle phase (Boulet et al., 2000, Mansoori et al., 2002, 2005, Chagras et al., 2005), population balance models (Mihálykó et al., 2004, Lakatos et al., 2006, 2008), and CFD simulation in the framework of EulerianEulerian approach (Chang and Yang, 2010) have been applied.

The population balance equation is a widely used tool in modeling the disperse systems of process engineering (Ramkrishna, 2000) describing a number of fluid-particle and particleparticle interactions. This equation was extended by Lakatos et al. (2006) with terms describing also the direct exchange processes of extensive quantities, such as mass and heat between the disperse elements as well as between the disperse elements and solid surfaces by collisional interactions (Lakatos et al., 2008).

The population balance model for describing the collisional particle-particle and particlesurface heat transfers was developed on the basis of a spatially homogeneous perfectly mixed system (Lakatos et al., 2008). In order to take into consideration also the spatial inhomogeneities of particles in a processing system a compartment/population balance model was introduced (Süle et al., 2006) which has proved applicable to model turbulent fluidization and the gas-solid fluidized bed heat exchangers (Süle et al., 2008). However, the spatial transport of gas and particles in turbulent fluidized beds usually is modeled by continuous dispersion models (Bi et al., 2000) thus, in order to achieve easier correlations of the constitutive variables, it has appeared reasonable to formulate the population balance combining it with the axial dispersion model (Süle et al., 2009 2010).

Particle-particle and particle-wall heat transfers may result from three mechanisms: heat transfers by radiation, heat conduction through the contact surface between the collided bodies, and heat transfers through the gas lens at the interfaces between the particles, as well as between the wall and particles collided with that. Heat conduction through the contact surface was modeled by Schlünder (1984), Martin (1984) and Sun and Chen (1988) developing analytical expressions for particle-particle and particle-wall contacts. Often, however, the conductive heat exchange can hardly be isolated from the mechanism 
occurring through the gas lens at the interfaces of the colliding bodies. Based on this mechanism, Vanderschuren and Delvosalle (1980) and Delvosalle and Vanderschuren (1985) developed a deterministic model for describing particle-particle heat transfer, while a model for heat transfer through the gas lens between a surface and particles was derived by Molerus (1997). Mihálykó et al. (2004) and Lakatos et al. (2008), based on the assumption that most factors characterizing the simultaneous heat transfer through the contact point and the gas lens are stochastic quantities described the collisional interparticle heat transfer by means of an aggregative random parameter.

In this chapter, a generalized population balance model is formulated to analyze heat transfer processes in gas-solid processing systems with inter-particle and particle-wall interactions by collisions, taking into consideration the thermal effects of collisions and the gassolid, gas-wall and wall-environment heat transfers. The population balance equation is developed for describing the spatial variation of temperature distribution of the particle population, and that of the gas and wall. The heat effects of energy change by collisions are included as a heat source in the particles. An infinite hierarchy of moment equations is derived, and a second order moment equation model is applied to analyze the thermal properties and behavior of bubbling fluidization by simulation.

\section{The population balance approach}

Consider a large population of solid particles being in intensive, turbulent motion in the physical space of a process vessel under the influence of some gas carrier. If the particulate phase is dense then particle collisions show significant effects on the behavior of the system therefore particle-particle and particle-surface heat transfer by collisions also may play important role in forming the thermal properties of system.

Let us assume that follow.

1) The two phase system is operated under developed hydrodynamic conditions.

2) Particles are mono-disperse and their size does not change during the course of the process.

3) Only thermal processes occur in the system without any mass transfer effects.

4) The temperature inside a particle can be taken homogeneous.

5) Heat transfer between the gas and particles, the wall and gas, as well as the wall and environment of the process vessel are continuous processes modeled by linear forces.

6) Heat transfer by radiation is negligible.

Under such conditions the state of a particle at time $t$ is given by the vector $\left(\mathbf{x}_{p}, \mathbf{u}_{p}, T_{p}\right)$ where $\mathbf{x}_{p}$ denote the space coordinates, $\mathbf{u}_{p}$ are the velocities along the space coordinates, and $T_{p}$ stands for the temperature of particles. The space coordinates, according to the nomenclature of population balance approach (Lakatos et al., 2006) are external properties of particles, while the particle velocities and temperature are internal ones.

Since a dense gas-solid system consists of a sufficient number of particles in the vicinity of each space coordinate $x$ therefore discontinuities can be smoothed out by introducing the population distribution function $(\mathbf{x}, \mathbf{u}, T, t) \rightarrow N(\mathbf{x}, \mathbf{u}, T, t)$ by the following

Definition. Let $\hat{N}(\mathbf{x}, \mathbf{u}, T, t)$ be a monotone non-decreasing function such that for every integrable and bounded function $g: X \rightarrow R$ the equality

$$
\int_{\hat{\boldsymbol{x}}} g(\mathbf{x}, \mathbf{u}, T) \hat{N}(d \mathbf{x}, d \mathbf{u}, d T, t)=\sum_{n=1}^{n=N(t)} g\left(\mathbf{x}_{p}^{n}, \mathbf{u}_{p}^{n}, T_{p}^{n}\right),\left(\mathbf{x}_{p}^{n}, \mathbf{u}_{p}^{n}, T_{p}^{n}\right) \in \hat{\boldsymbol{X}}
$$


holds where the triplet $\left(\mathbf{x}_{p}^{n}, \mathbf{u}_{p}^{n}, T_{p}^{n}\right)$ denotes the space coordinates, velocities and temperature of the $n^{\text {th }}$ particle, $\hat{\boldsymbol{x}}$ stands for the state space of coordinates, velocities and temperature, and $N(t)$ is the total number of particles in a unit volume at the moment of time $t$.

For practical reasons, instead of the population distribution function usually the population density function $(\mathbf{x}, \mathbf{u}, T, t) \rightarrow \hat{n}(\mathbf{x}, \mathbf{u}, T, t)$ is applied that is determined as

$$
\hat{n}(\mathbf{x}, \mathbf{u}, T, t)=\frac{\partial^{7} \hat{N}(\mathbf{x}, \mathbf{u}, T, t)}{\partial \mathbf{x} \partial \mathbf{u} \partial T}
$$

by means of which $\hat{n}(\mathbf{x}, \mathbf{u}, T, t) d \mathbf{x} d \mathbf{u} d T$ denotes the number of particles in the region $V(\mathbf{x}, \delta \mathbf{x})$ of physical space from the domain $V(\mathbf{u}, \delta \mathbf{u})$ of velocity and interval of temperature $(T, T+d T)$ at time $t$.

From the density function $\hat{n}(\mathbf{x}, \mathbf{u}, T, t)$ we obtain a reduced population density function

$$
n(\mathbf{x}, T, t)=\int_{\mathbf{U}} \hat{n}(\mathbf{x}, \mathbf{u}, T, t) d \mathbf{u}
$$

by means of which $n(\mathbf{x}, T, t) d \mathbf{x} d T$ denotes the number of particles of all velocities in the region $V(\mathbf{x}, \delta \mathbf{x})$ of physical space and interval of temperature $(T, T+d T)$ at time $t$.

The population density functions $\hat{n}(., \ldots, t)$ and $n(.,, t)$, depending on the practical reasons, are interpreted as the states of particle populations.

\section{Population balance equation}

\subsection{Integral forms with transition measures}

According to the assumptions of former section particles are in intensive, stochastic motion in some domain $X \subseteq R^{7}$ of the metric space $R^{7}$ of external and internal properties therefore the time variation of particle state is described by the set of stochastic differential equations

$$
\begin{gathered}
d \mathbf{x}_{p}(t)=\mathbf{u}_{p}(t) d t \\
m_{p} d \mathbf{u}_{p}(t)=-\alpha \mathbf{u}_{p}(t) d t+\sum \mathbf{f} d t+\mathbf{\sigma}_{\mathbf{u}, p} d \mathbf{W}(t)+\int_{\hat{\boldsymbol{x}}} \Xi_{\mathbf{u}_{p}}\left(\mathbf{u}_{p}\right) \boldsymbol{n}\left(t, d \mathbf{x}_{p}, d \mathbf{u}_{p}, d T_{p}\right) \\
m_{p} c_{p} d T_{p}(t)=\beta T_{p}(t) d t+\int_{\hat{\boldsymbol{x}}} \Xi_{T_{p}}\left(T_{p}\right) \boldsymbol{n}\left(t, d \mathbf{x}_{p}, d \mathbf{u}_{p}, d T_{p}\right)
\end{gathered}
$$

subject to the appropriate initial conditions. In Eqs (3)-(5), $\sum \mathbf{f}$ are deterministic forces while $\mathbf{W}(t)$ is a Wiener process, inducing motion of particles in the physical space, $a$ and $\beta$ are deterministic functions characterizing the continuous motion in the physical and temperature subspace, while the integrals in Eqs (4) and (5) describe jump-like stochastic changes of internal properties. Here, function $\boldsymbol{n}_{(.)}$determines the conditions of collisions of particles, while functions $\Xi_{\mathbf{u}_{p}}\left(\mathbf{u}_{p}\right)$ and $\Xi_{T_{p}}\left(T_{p}\right)$ denote, respectively, the velocity and temperature jumps induced by those.

The set of stochastic differential equations (3)-(5) describes the behavior of the population of particles entirely by tracking the time evolution of the state of each particle individually. However, numerical solution of Eqs (3)-(5) is a crucial problem although it would provide 
detailed information about the life of each particle. Monte Carlo simulation means a good method for solving this system but to generate realistic results rather a large sample of particle population is required leading to long computer times.

However, for engineering purposes we usually are interested only in the behavior of the particle population as a whole. Naturally, this information would be generated also by solving the system of stochastic differential equations (3)-(5) but we can obtain it directly applying the population balance approach. Namely, taking into consideration that the system of stochastic equations (3)-(5) induces a Markov process $\left\{\mathbf{x}_{p}(t), \mathbf{u}_{p}(t), T_{p}(t)\right\}_{t \geq 0}$ having continuous sample paths with finite jumps (Gardiner,1983, Sobczyk,1991), the particulate system exhibits all the properties of interactive disperse systems (Lakatos et al., 2006) for which we can derive a conditional transition measure $\hat{P}_{c}$ by means of which the variation of the state of population of particles is described by the transformation

$$
\begin{array}{r}
\hat{n}(\mathbf{x}, \mathbf{u}, T, t)=\frac{1}{N(s)} \int_{\hat{\mathbf{x}}} \int_{\hat{\boldsymbol{x}}} \hat{P}_{c}\left(s, \mathbf{x}^{\prime}, \mathbf{u}^{\prime}, T^{\prime} ; t, \mathbf{x}, \mathbf{u}, T \mid \mathbf{x}^{\prime \prime}, \mathbf{u}^{\prime \prime}, T^{\prime \prime}\right) \times \\
\times \hat{n}\left(\mathbf{x}^{\prime}, \mathbf{u}^{\prime}, T^{\prime}, s\right) \hat{n}\left(\mathbf{x}^{\prime \prime}, \mathbf{u}^{\prime \prime}, T^{\prime \prime}, s\right) d \mathbf{x}^{\prime} d \mathbf{x}^{\prime \prime} d \mathbf{u}^{\prime} d \mathbf{u}^{\prime \prime} d T^{\prime} d T^{\prime \prime} \\
t>s ;(\mathbf{x}, \mathbf{u}, T) \in \hat{\boldsymbol{X}}
\end{array}
$$

where $N(s)$ denotes the number of particles in the given domain at time $s$

$$
N(s)=\int_{\hat{\boldsymbol{x}}} \hat{n}(\mathbf{x}, \mathbf{u}, T, s) d \mathbf{x} d \mathbf{u} d T .
$$

In Eq.(6), expression

$$
\frac{1}{N(s)} \hat{n}\left(\mathbf{x}^{\prime \prime}, \mathbf{u} ", T^{\prime \prime}, s\right) d \mathbf{x} " d \mathbf{u} " d T "
$$

is interpreted as the probability that there exists a solid particle in the state domain $\left(\mathbf{x}^{\prime \prime}, \mathbf{u}^{\prime \prime}, T^{\prime \prime}, \mathbf{x}^{\prime \prime}+d \mathbf{x}^{\prime \prime}, \mathbf{u}^{\prime \prime}+d \mathbf{u}^{\prime \prime}, T^{\prime \prime}+d T^{\prime \prime}\right)$ possibly interacting with a particle of state $\left(\mathbf{x}^{\prime}, \mathbf{u}^{\prime}, T^{\prime}\right)$ and the result of this interaction event is expressed by the conditional transition measure $\hat{P}_{c}$. The properties of the conditional transition measure $\hat{P}_{c}$ are determined by the physicalchemical processes of the system that induce motion and/or formation of particles under given operational conditions. When the action of these processes can be described by means of some vector of random parameters $\theta$ with distribution function $F_{\theta}($.$) , and all particles are$ moved and/or formed under the same conditions then a lot of different realisations, described by the distribution function of the random parameters $\theta$, can act on the particles. As a result, the final population is formed as expectation for the vector of parameters $\boldsymbol{\theta}$ so that Eq.(6) can be rewritten using randomization:

$$
\begin{gathered}
\hat{n}(\mathbf{x}, \mathbf{u}, T, t)=\frac{1}{N(s)} \iint_{\Theta} \int_{\hat{\mathbf{x}}} \tilde{P}_{c}\left(s, \mathbf{x}^{\prime}, \mathbf{u}^{\prime}, T^{\prime} ; t, \mathbf{x}, \mathbf{u}, \mathbf{c} \mid \mathbf{x}^{\prime \prime}, \mathbf{u}^{\prime \prime}, T^{\prime \prime}, \boldsymbol{\theta}\right) \times \\
\times \hat{n}\left(\mathbf{x}^{\prime}, \mathbf{u}^{\prime}, T^{\prime}, s\right) \hat{n}\left(\mathbf{x}^{\prime \prime}, \mathbf{u}^{\prime \prime}, T^{\prime \prime}, s\right) d \mathbf{x}^{\prime} d \mathbf{x}^{\prime \prime} d \mathbf{u}^{\prime} d \mathbf{u}^{\prime \prime} d T^{\prime} d T^{\prime \prime} F_{\boldsymbol{\theta}}(d \boldsymbol{\theta}) \\
t>s ;(\mathbf{x}, \mathbf{u}, T) \in \hat{\boldsymbol{X}}
\end{gathered}
$$


Eq.(9) is, in principle, a multidimensional, i.e. $(3+4) \mathrm{D}$ population balance equation written in integral form by means of the transition measure defined on the basis of transition probability of Markov processes. Here, indicating the dimension of the equation the first number denotes the external variables while the second one gives number of internal variables. The parameterized conditional transition measure $\tilde{P}_{c}$ of the particulate system involves, in principle, all information about the properties and behavior of the particle population and precision of the nature and components of motions along the property coordinates of population state makes possible to derive the corresponding population balance equation. However, this multidimensional population balance equation seems to be too complex; simplification is obtained by deriving a population balance equation for the ensemble of particles of all velocities

$$
n(\mathbf{x}, T, t)=\int_{\mathbf{U}} \hat{n}(\mathbf{x}, \mathbf{u}, T, t) d \mathbf{u}
$$

modeling motion of particles in the physical space by using convection-dispersion models. Rewriting the population density function in the conditional form

$$
\frac{1}{N(s)} \hat{n}(\mathbf{x}, \mathbf{u}, T, t)=\operatorname{Prob}(\mathbf{u} \mid \mathbf{x}, T) \frac{1}{N(s)} n(\mathbf{x}, T, t)
$$

where $\operatorname{Prob}(\mathbf{u} \mid \mathbf{x}, T)$ denotes the probability that a particle, residing at space coordinate $x$ and having temperature $T$ possesses velocity $\boldsymbol{u}$, introducing (11) into Eq.(9) and integrating both sides of Eq.(9) over variable $\boldsymbol{u}$ we obtain

$$
\begin{gathered}
n(\mathbf{x}, T, t)=\frac{1}{N(s)} \int_{\boldsymbol{X}} \int_{\boldsymbol{X}} P_{c}\left(s, \mathbf{x}^{\prime}, T^{\prime} ; t, \mathbf{x}, T \mid \mathbf{x}^{\prime \prime}, T^{\prime \prime}, \boldsymbol{\theta}\right) \times \\
\times n\left(\mathbf{x}^{\prime}, T^{\prime}, s\right) n\left(\mathbf{x}^{\prime \prime}, T^{\prime \prime}, s\right) d \mathbf{x}^{\prime} d \mathbf{x}^{\prime \prime} d T^{\prime} d T^{\prime \prime} F_{\boldsymbol{\theta}}(d \boldsymbol{\theta}) \\
t>s ;(\mathbf{x}, T) \in \mathbf{X}
\end{gathered}
$$

where

$$
\begin{aligned}
& P_{c}\left(s, \mathbf{x}^{\prime}, T^{\prime} ; t, \mathbf{x}, T \mid \mathbf{x}^{\prime \prime}, T^{\prime \prime}, \boldsymbol{\theta}\right)=\iint_{U} \int_{U} \tilde{P}_{\mathcal{U}}\left(s, \mathbf{x}^{\prime}, \mathbf{u}^{\prime}, T^{\prime} ; t, \mathbf{x}, \mathbf{u}, T \mid \mathbf{x}^{\prime \prime}, \mathbf{u}^{\prime \prime}, T^{\prime \prime}, \boldsymbol{\theta}\right) \times \\
& \times \operatorname{Prob}\left(\mathbf{u}^{\prime} \mid \mathbf{x}^{\prime}, T^{\prime}\right) \operatorname{Prob}\left(\mathbf{u}^{\prime \prime} \mid \mathbf{x}^{\prime \prime}, T^{\prime \prime}\right) d \mathbf{u}^{\prime} d \mathbf{u}^{\prime \prime} d \mathbf{u}
\end{aligned}
$$

Here, expression (13) denotes the transition measure by means of which transformation (12) provides the amount of particles of all velocities which are converted into the state domain $(\mathbf{x}, T, \mathbf{x}+d \mathbf{x}, T+d T)$ to time $t$ of particles of all velocities being in $\hat{\boldsymbol{x}}$ at time $s$ under the conditions of interactions with particles of the same state domain of any velocities. As a consequence, transformation (12) describes motion of particles with possible heat exchange interactions in the $3+1$ dimensional subspace of physical and temperature coordinates determined over a given, fully developed velocity field. Eq.(12) is a reduced form of the multidimensional population balance equation (9), given also in integral form. 


\subsection{Integral-differential equation form}

Taking into account the conditions assumed and following the steps of derivation of the population balance equation of interactive disperse systems as it was presented by Lakatos et al.(2006), the population balance equation describing the behavior of population of the particles in the system is obtained from Eq.(9) in the form

$$
\begin{aligned}
& \frac{\partial \hat{n}(\mathbf{x}, \mathbf{u}, T, t)}{\partial t}+\frac{\partial}{\partial T}\left[G_{T} \hat{n}(\mathbf{x}, \mathbf{u}, T, t)\right]+\nabla_{\mathbf{u}} \cdot\left[\mathbf{G}_{\mathbf{u}} \hat{n}(\mathbf{x}, \mathbf{u}, T, t)\right]= \\
& =\nabla_{\mathbf{x}} \cdot \mathbf{D}_{\mathbf{x}} \nabla_{\mathbf{x}} \hat{n}(\mathbf{x}, \mathbf{u}, T, t)-\nabla_{\mathbf{x}} \cdot\left[\mathbf{G}_{\mathbf{x}} \hat{n}(\mathbf{x}, \mathbf{u}, T, t)\right]- \\
& -\frac{\hat{n}(\mathbf{x}, \mathbf{u}, T, t)}{N(\mathbf{x}, t)} \int_{U} \int_{T_{\min }}^{T_{\max }} \hat{S}_{p p}\left(T, \mathbf{u} \mid T^{\prime}, \mathbf{u}^{\prime}\right) \hat{n}\left(\mathbf{x}, \mathbf{u}^{\prime}, T^{\prime}, t\right) d T^{\prime} d \mathbf{u}^{\prime}+ \\
& +\frac{1}{N(\mathbf{x}, t)} \iint_{\Theta} \int_{\boldsymbol{U}} \int_{T_{\min }}^{T_{\max }} \int_{T_{\min }}^{T_{\max }} \hat{S}_{p p}\left(T^{\prime}, \mathbf{u}^{\prime} \mid T^{\prime \prime}, \mathbf{u}^{\prime \prime}\right) \hat{b}_{p p}\left(T^{\prime}, T, \mathbf{u}^{\prime}, \mathbf{u} \mid T^{\prime \prime}, \mathbf{u}^{\prime \prime}, \boldsymbol{\theta}\right) \times \\
& \times \hat{n}\left(\mathbf{x}, \mathbf{u}^{\prime}, T^{\prime}, t\right) \hat{n}\left(\mathbf{x}, \mathbf{u}^{\prime \prime}, T^{\prime \prime}, t\right) d T^{\prime} d T^{\prime \prime} d \mathbf{u}^{\prime} d \mathbf{u}^{\prime \prime} \hat{F}_{\boldsymbol{\theta}}(d \boldsymbol{\theta})
\end{aligned}
$$

subject to the appropriate boundary and initial conditions. Here, $N(\mathbf{x}, t)$ denotes the total number of particles in the vicinity of position $x$ of the physical space at the moment of time $t$.

The second and third terms on the left hand side of Eq.(14), next to the accumulation term describe variation of the population density function $\hat{n}$ due to continuous thermal and velocity interactions of particles with the carrier gas environment, respectively, while the first and second terms on the right hand side describe motion of particles in the physical space. Here, the rate expressions $G_{T}, \mathbf{G}_{\mathbf{u}}$ and $\mathbf{G}_{\mathbf{x}}$ are interpreted as convective terms of motion along the temperature, velocity and physical coordinates, and $\mathbf{D}_{\mathbf{x}}$ denotes the dispersion tensor of motion of particles in the physical space.

The last two terms on the right hand side of Eq.(14) describe the momentum and heat exchange between particles interacting with each other by collisions during their motion in the physical space. Namely, the third term describe the rate of decrease of number of particles of temperature $T$ and velocity $\boldsymbol{u}$ collided with particles of temperature $T^{\prime}$ and velocity $\boldsymbol{u}^{\prime}$, whereas the last term provides the rate of increase of number of particles having temperature $T$ and velocity $u$ resulted in collision interactions of particles of temperature $T^{\prime}$ and $T^{\prime \prime}$ as well as of velocity $\boldsymbol{u}^{\prime}$ and $\boldsymbol{u}^{\prime \prime}$. The collision interactions are described by means of a product of two operators: the activity and conversion functions $\hat{S}_{p p}$ and $\hat{b}_{p p}$, respectively. In the present model, the activity function $\hat{S}_{p p}$ characterizes the intensity of collision interactions between the particles inducing temperature change, being, in turn, a product of the frequency of collisions $\hat{S}_{c o l}\left(T, \mathbf{u} \mid T^{\prime}, \mathbf{u}^{\prime}\right)$ and the efficiency term $\hat{S}_{e x}\left(T, \mathbf{u} \mid T^{\prime}, \mathbf{u}^{\prime}\right)$, written as $\hat{S}_{p p}\left(T, \mathbf{u} \mid T^{\prime}, \mathbf{u}^{\prime}\right)=\hat{S}_{c o l}\left(T, \mathbf{u} \mid T^{\prime}, \mathbf{u}^{\prime}\right) \cdot \hat{S}_{e x}\left(T, \mathbf{u} \mid T^{\prime}, \mathbf{u}^{\prime}\right)$. Here, $\hat{S}_{e x}\left(T, \mathbf{u} \mid T^{\prime}, \mathbf{u}^{\prime}\right)$ expresses the ratio of all events which are to be successful in inducing also heat and momentum exchange events. Naturally, since heat diffusion, deciding at this level on homogenization of temperature is a spontaneous process, while momentum exchange by collision of two solid bodies also occurs the efficiency term is equal to unity identically, i.e. $\hat{S}_{e x}\left(T, \mathbf{u} \mid T^{\prime}, \mathbf{u}^{\prime}\right) \equiv 1$. Finally, function $\hat{b}_{p p}\left(T^{\prime}, T, \mathbf{u}^{\prime}, \mathbf{u} \mid T^{\prime \prime}, \mathbf{u}^{\prime \prime}, \boldsymbol{\theta}\right)$, called conversion density function, describes the results of events inducing changes of temperature and velocity of particles, i.e. $\hat{b}_{p p}\left(T^{\prime}, T, \mathbf{u}^{\prime}, \mathbf{u} \mid T^{\prime \prime}, \mathbf{u}^{\prime \prime}, \boldsymbol{\theta}\right) d T d \mathbf{u}$ expresses the fraction of particles that become of temperature 
$(T, T+d T)$ and $(\mathbf{u}, \mathbf{u}+d \mathbf{u})$ as a result of interactions of all particles of temperature $T^{\prime}$ and $T^{\prime \prime}$ and velocity $\boldsymbol{u}^{\prime}$ and $\boldsymbol{u}^{\prime \prime}$ in a unit time.

The $(3+4)$ dimensional population balance equation (14) is a so called cognitive model that describes the behavior of particle population in detail taking into account also the velocity distribution of particles. However, a simplified population balance equation can be obtained from Eq.(12) for the population density function $n(\mathbf{x}, T, t)$ not including the explicit velocity dependence into the model. Indeed, again following the steps of derivation of the population balance equation of interactive disperse systems we obtain

$$
\begin{aligned}
& \frac{\partial n(\mathbf{x}, T, t)}{\partial t}+\frac{\partial}{\partial T}\left[G_{T} n(\mathbf{x}, T, t)\right]=\nabla_{\mathbf{x}} \cdot \mathbf{D}_{\mathbf{x}} \nabla_{\mathbf{x}} n(\mathbf{x}, T, t)-\nabla_{\mathbf{x}} \cdot\left[\mathbf{G}_{\mathbf{x}} n(\mathbf{x}, T, t)\right] \\
& -\frac{n(\mathbf{x}, T, t)}{N(\mathbf{x}, t)} \int_{T_{\min }}^{T_{\max }} S_{p p}\left(T \mid T^{\prime}\right) n\left(\mathbf{x}, T^{\prime}, t\right) d T^{\prime}+ \\
& +\frac{1}{N(\mathbf{x}, t)} \int_{\Theta}^{T_{\max }} \int_{T_{\min }}^{T_{\max }} b_{T_{\min }}\left(T^{\prime}, T \mid T^{\prime \prime}, \boldsymbol{\theta}\right) S_{p p}\left(T^{\prime} \mid T^{\prime \prime}\right) n\left(\mathbf{x}, T^{\prime}, t\right) n\left(\mathbf{x}, T^{\prime \prime}, t\right) d T^{\prime} d T^{\prime \prime} F_{\boldsymbol{\theta}}(d \boldsymbol{\theta})
\end{aligned}
$$

subject to the appropriate boundary and initial conditions. Here, the constitutive expressions in Eq.(15) have similar meanings as it was described regarding Eq.(14) excluding the explicit velocity dependence. Note that in this simplified form the effects of collisions on motion of particles in the physical space can be taken into consideration by applying empirical models for the convective and dispersion terms $\mathbf{G}_{\mathbf{x}}$ and $\mathbf{D}_{\mathbf{x}}$, respectively. In the context of the population balance equation (15), the particle-solid surface boundary condition is relevant that can be formulated as the heat exchange rate between the particle population and solid surface at a space coordinate $x$ of surface $S$

$$
\begin{aligned}
& \left.d Q_{w p}(\mathbf{x}, t)\right|_{\mathcal{S}}=-\left.\int_{\Theta} \int_{T_{\min }}^{T_{\max }} S_{w p}\left(T_{w}(\mathbf{x}, t) \mid T\right) b_{w p}\left(T_{w}(\mathbf{x}, t), z \mid T, \boldsymbol{\theta}\right) n(\mathbf{x}, z, t) d z F_{\boldsymbol{\theta}}(d \boldsymbol{\theta})\right|_{\mathcal{S}} d t+ \\
& +\left.\int_{\boldsymbol{\Theta}} \int_{T_{\min }}^{T_{\max }} S_{w p}\left(T_{w}(\mathbf{x}, t) \mid z\right) b_{w p}\left(T_{w}(\mathbf{x}, t), T \mid z, \boldsymbol{\theta}\right) n(\mathbf{x}, z, t) d z F_{\boldsymbol{\theta}}(d \boldsymbol{\theta})\right|_{\mathcal{S}} d t
\end{aligned}
$$

where $T_{w}(x, t)$ denotes the temperature of surface $S$ at position $x$ at time $t$.

Eq.(15) with the boundary condition is an applicative or, in other words, a purpose-oriented partially distributed population balance model aimed for describing thermal processes in fluid-solid particulate systems. Introducing the constitutive expressions and making use of appropriate symmetric conditions it can be applied for determining spatially low dimensional population balance models. Here, the one-dimensional axial dispersion/population balance and the zero-dimensional well stirred vessel/population balance model will be presented to describe thermal processes in gas-solid particulate systems.

\section{Constitutive equations for particle-particle heat transfer by collisions}

\subsection{Heat effects of particle-particle collisions}

To close the population balance equation (15) the constitutive equations, first of all those characterizing the collision interactions are to be determined. In developing we consider 
only binary collisions as it is shown schematically in Figs 1 and 2. Here we assumed that particles in the particulate system are of the same volume and mass but their shape usually are irregular. As a consequence, in this case the collision events are of random nature therefore practically all processes induced by collisions, among others the change of kinetic energy and exchange of extensive quantities between the colliding particles should be treated as random.

Let us now consider the encounter of two solid particles of mass $m_{j}$ and $m_{k}$ as it is shown in Fig.1. If the velocity vectors of these bodies are given by $\boldsymbol{u}_{j}$ and $\boldsymbol{u}_{k}$, and their difference is denoted by $\boldsymbol{u}_{j k}=\boldsymbol{u}_{j}-\boldsymbol{u}_{k}$ then the translational kinetic energy change during collision $\Delta E$ is

$$
\Delta E=\frac{1}{2} \frac{m_{j} m_{k}}{m_{j}+m_{k}}\left(u_{j k}^{2}{ }^{\prime}-u_{j k}^{2}\right)=\frac{1}{2} \frac{m_{j} m_{k}}{m_{j}+m_{k}}\left(e^{2}-1\right) u_{j k}^{2}
$$

where $e$ is assumed to be a random coefficient of restitution. If the two particles are of equal mass, i.e. $m_{j}=m_{k}=m_{p}$ then

$$
\Delta E=\frac{m_{p}}{4}\left(e^{2}-1\right) u_{j k}^{2}
$$

and accounting for the probability that a particle is colliding with an another particle in the time interval $(t, t+\Delta t)$ the energy change is expressed as

$$
\Delta E(\mathbf{x}, t)=\frac{m_{p}\left(e^{2}-1\right)}{4 N(\mathbf{x}, t)} \iint_{\mathbf{U} \times T} u_{j k}^{2} \hat{n}\left(\mathbf{x}, \mathbf{u}_{j k}, T, t\right) d \mathbf{u}_{j k} d T \cdot \Delta t
$$

Applying the Maxwellian distribution for particles

$$
\hat{n}\left(\mathbf{x}, \mathbf{u}_{j k}, T, t\right)=\frac{n(\mathbf{x}, T, t)}{\left(\frac{2}{3} \pi\left\langle\mathbf{u}^{2}\right\rangle\right)^{\frac{3}{2}}} \exp \left(-\frac{\mathbf{u}_{j k}^{2}}{\frac{2}{3}\left\langle\mathbf{u}^{2}\right\rangle}\right)
$$

and taking into consideration that the coefficient of restitution is a random variable with the probability density function $f_{e}$, independent from the velocities, the mean value of energy change can be expressed as

$$
\Delta E(\mathbf{x}, t)=\frac{m_{p} \int_{0}^{1}\left(e^{2}-1\right) f_{e}(e) d e}{4 N(\mathbf{x}, t)\left(\frac{2}{3} \pi\left\langle\mathbf{u}^{2}\right\rangle\right)^{\frac{3}{2}}} \iint_{\boldsymbol{U} \times T} n(\mathbf{x}, T, t) u_{j k}^{2} \exp \left(-\frac{\mathbf{u}_{j k}^{2}}{\frac{2}{3}\left\langle\mathbf{u}^{2}\right\rangle}\right) d \mathbf{u}_{j k} d T \cdot \Delta t .
$$

With complete spherical symmetry

$$
d \mathbf{u}_{j k}=4 \pi u_{j k}^{2} d u_{j k}
$$


we have

$$
\Delta E(\mathbf{x}, t)=\frac{4 \pi m_{p} \int_{0}^{1}\left(e^{2}-1\right) f_{e}(e) d e}{4 N(\mathbf{x}, t)\left(\frac{2}{3} \pi\left\langle\mathbf{u}^{2}\right\rangle\right)^{\frac{3}{2}}} \iint_{U \times T} n(\mathbf{x}, T, t) u_{j k}^{4} \exp \left(-\frac{\mathbf{u}_{j k}^{2}}{\frac{2}{3}\left\langle\mathbf{u}^{2}\right\rangle}\right) d u_{j k} d T \cdot \Delta t
$$

Making use of evaluation of the definite integral (Gidaspow, 1994)

$$
\int_{0}^{1} x^{4} \exp \left(-a x^{2}\right) d x=\frac{3 \sqrt{\pi}}{8} \alpha^{-\frac{5}{2}}
$$

we obtain

$$
\Delta E(\mathbf{x}, t)=\frac{m_{p}}{4 N(\mathbf{x}, t)}\left\langle\mathbf{u}^{2}\right\rangle \int_{0}^{1}\left(e^{2}-1\right) f_{e}(e) d e \int_{T} n(\mathbf{x}, T, t) d T \cdot \Delta t
$$

As a consequence, assuming that this heat arise in particles as it was generated by an internal heat source we can write

$$
c_{p} m_{p} \frac{d T}{d t}=\frac{1}{2} \frac{d E(\mathbf{x}, t)}{d t}=\frac{m_{p}}{8}\left\langle\mathbf{u}^{2}\right\rangle \int_{0}^{1}\left(e^{2}-1\right) f_{e}(e) d e
$$

that results in the following rate of temperature change for a particle

$$
\frac{d T}{d t}=\frac{\left\langle\mathbf{u}^{2}\right\rangle}{8 c_{p}} \int_{0}^{1}\left(e^{2}-1\right) f_{e}(e) d e .
$$

\subsection{Heat transfer by collisions of two particles}

Let us consider the heat transfer process induced by collision of two solid bodies, shown schematically in Fig. 1, as described by the set of simple first order differential equations with linear driving forces

$$
m_{j} c_{j} \frac{d T_{j}(t)}{d t}=\beta\left(T_{k}(t)-T_{j}(t)\right)
$$

and

$$
m_{k} c_{k} \frac{d T_{k}(t)}{d t}=-\beta\left(T_{k}(t)-T_{j}(t)\right)
$$

subject to the initial conditions

$$
T_{j}(0)=T_{j 0}, \quad T_{k}(0)=T_{k 0}
$$


where $\beta$ is a not specified aggregated heat transfer coefficient.

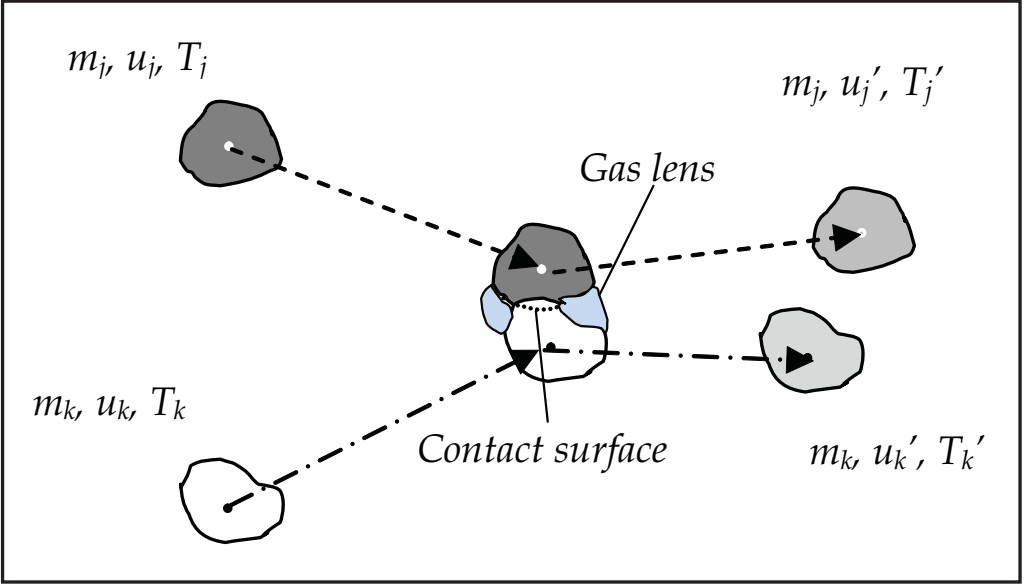

Fig. 1. Collision of two particles of irregular shape

Eqs (28) are of such forms as the temperature of particles would be uniform. However it is not the case so that these equations have to be interpreted as they were written for the mean values. In this case the variables are, in essence, mean values, i.e.

$$
T_{\imath}(t)=\frac{1}{v_{t}} \int T_{t} d V, \quad \imath=j, k
$$

Since the temperature of a particle is not uniform the actual temperature values in the driving force in Eq.(28) are uncertain so that this should be treated also as random. Therefore the left hand sides of Eq.(28) are random and we allocate this randomness into the $\beta$ transfer coefficient.

The solutions of the first order differential equations (28) at time $\theta$ become

$$
T_{j}(\theta)=T_{j 0}+\frac{m_{k} c_{k}}{m_{j} c_{j}+m_{k} c_{k}}\left(T_{k 0}-T_{j 0}\right)\left\{1-\exp \left[-\beta \theta\left(\frac{1}{m_{j} c_{j}}+\frac{1}{m_{k} c_{k}}\right)\right]\right\}=T_{j 0}+p_{j} \omega\left(T_{k 0}-T_{j 0}\right)
$$

and

$$
T_{k}(\theta)=T_{k 0}-\frac{m_{j} c_{j}}{m_{j} c_{j}+m_{k} c_{k}}\left(T_{k 0}-T_{j 0}\right)\left\{1-\exp \left[-\beta \theta\left(\frac{1}{m_{j} c_{j}}+\frac{1}{m_{k} c_{k}}\right)\right]\right\}=T_{k 0}-p_{k} \omega\left(T_{k 0}-T_{j 0}\right)
$$

where

$$
\omega:=1-\exp \left[-\beta \theta\left(\frac{m_{j} c_{j}+m_{k} c_{k}}{m_{j} c_{j} m_{k} c_{k}}\right)\right], p_{j}=\frac{m_{k} c_{k}}{m_{j} c_{j}+m_{k} c_{k}}, p_{k}=\frac{m_{j} c_{j}}{m_{j} c_{j}+m_{k} c_{k}}
$$


Here $\theta$ denotes the contact time which is, in principle, also a random quantity. As a consequence, parameter $\omega \in[0,1]$, characterizing the efficiency of heat exchange between the colliding bodies is a random function of variables $\beta$ and $\theta$, and its distribution is entirely determined by their distribution functions.

Let us now assume that the two bodies suffering collisions are two particles with equal masses and heat capacities. Then, $m_{j} c_{j}=m_{k} c_{k}=m_{p} c_{p}$ and $p_{j}=p_{k}=\frac{1}{2}$. Introducing the notation $T_{j 0}=T_{p}{ }^{\prime}, T_{k 0}=T_{p}{ }^{\prime \prime}$ we can write

$$
\omega=\omega_{p p}=1-\exp \left[\frac{-2 h_{p p} a_{p p} \theta_{p p}}{m_{p} C_{p}}\right]
$$

and

$$
T_{p}(\theta)=T_{p}{ }^{\prime}+\left(T_{p}{ }^{\prime \prime}-T_{p}{ }^{\prime}\right) \cdot \frac{\omega_{p p}}{2}
$$

from which

$$
\frac{2\left(T_{p}-T_{p}^{\prime}\right)}{\omega_{p p}}+T_{p}{ }^{\prime}=T_{p}{ }^{\prime \prime}
$$

Interpretation of Eq.(12) is that in heat transfer process characterized with parameter $\omega_{p p}$ a particle with temperature $T_{p}{ }^{\prime}$ has to collide with particle of temperature $T_{p}{ }^{\prime \prime}$ to achieve final temperature $T_{p}$. Taking now into consideration the definitions of conversion functions, the conversion distribution function of particle-particle heat transfer takes the form

$$
B_{p p}\left(T_{p}{ }^{\prime}, T_{p} \mid T_{p}{ }^{\prime \prime}, \omega_{p p}\right)=1_{T_{p}}\left[\left(\frac{2\left(T_{p}-T_{p}^{\prime}\right)}{\omega_{p p}}+T_{p}{ }^{\prime}\right)-T_{p}{ }^{\prime}\right]
$$

so that the corresponding density function is

$$
b_{p p}\left(T_{p}{ }^{\prime}, T_{p} \mid T_{p}{ }^{\prime \prime}, \omega_{p p}\right) d T_{p}=\delta_{T_{p}}\left[\left(\frac{2\left(T_{p}-T_{p}{ }^{\prime}\right)}{\omega_{p p}}+T_{p}{ }^{\prime}\right)-T_{p}{ }^{\prime \prime}\right] \frac{2}{\omega_{p p}} d T_{p}
$$

\subsection{Heat transfer through the gas lens and the contact surface}

Heat transfer between two particles may be considered as a combination of transfers through the gas lens between the particles and through the contact surface as it is shown in Fig. 1 . These processes depend strongly on the shapes and velocities of the colliding bodies that is why the parameters $\beta$ and $\theta$ are treated as random variables. However, under symmetrical conditions for particles of regular spherical forms explicit expressions have been determined. 
The heat flow rate through the gas lens between two particles with diameter $d_{p}$ in the angle $\left(-\alpha_{\max },+\alpha_{\max }\right)$ was developed by Delvosalle and Vanderschuren (1985) in the form

$$
q_{h}=\pi h d_{p}\left(T_{k}-T_{j}\right) \int_{0}^{\alpha_{\max }} \frac{\sin ^{2} \alpha d \alpha}{2\left[\frac{s}{R}+(1-|\cos \alpha|)\right] \alpha}
$$

where $2 s$ denotes the width of the gas lens. At this flow rate the temperature of the $j^{\text {th }}$ particle during the time interval $(0, \theta)$ increases to the value

$$
T_{j}=T_{j 0}+\Gamma(\theta)\left(T_{k 0}-T_{j 0}\right)
$$

where

$$
\Gamma(\theta)=1-\exp \left(\frac{3 h}{\rho_{p} c_{p} d_{p}^{2}} \int_{0}^{\alpha_{\max }} \frac{\sin ^{2} \alpha d \alpha}{2\left[\frac{s}{R}+(1-|\cos \alpha|)\right] \alpha} \theta\right)
$$

In the case of intensive motion of particles, the parameters $h, s$ and $a_{\max }$ in Eq.(38) as well as the contact time $\theta$ are, in principle, random quantities therefore $\Gamma$ is also a random function of these arguments.

The heat flow rate through the contact surface was studied by Sun and Chen (1988) developing an approximate expression in the form

$$
q_{h}=\frac{\sqrt{\rho_{p} c_{p} k_{p}} t^{-\frac{1}{2}}}{2 \sqrt{\pi}}\left(T_{k}-T_{j}\right)
$$

by means of which the total temperature increase in the $j$ th particle during the time interval $(0, \theta)$ is expressed as

$$
T_{j}(\theta)=T_{j 0}+\frac{0.87 \sqrt{\rho_{p} c_{p} k_{p}} a_{c} \theta^{\frac{1}{2}}}{2 m_{p} c_{p}}\left(T_{k 0}-T_{j 0}\right)
$$

where $a_{c}$ denotes the maximum contact area. Again, in Eq.(42) the maximum contact area and contact time depend on the velocities of collided particles so that these quantities are to be treated also as random variables.

Comparing the equations (31), (39) and (42) all these are expressed by linear driving forces so that the overall heat transfer flow can be given as superposition of the heat flows through the gas lens and the contact surface.

\section{Constitutive equations for particle-wall heat transfer by collisions}

\subsection{Heat effects of particle-wall collisions}

Let us consider again the encounter of two solid bodies of mass $m_{j}$ and $m_{k}$ as it is shown in Fig. 2 from which now the second is a no moving solid surface. In this case the velocity 
vector $\boldsymbol{u}_{k}=0$ so that the velocity difference is $\boldsymbol{u}_{j k}=\boldsymbol{u}_{j}$ then the translational kinetic energy change $\Delta E$ is given as

$$
\Delta E=\frac{1}{2} \frac{m_{p} m_{w}}{m_{p}+m_{w}}\left(u_{j}^{2}-u_{j}^{2}\right)=\frac{m}{2} u_{j}^{2} \int_{0}^{1}\left(e^{2}-1\right) f_{e}(e) d e
$$

where, again, $e$ is a random coefficient of restitution and $m=m_{p} m_{w} /\left(m_{p}+m_{w}\right)$.

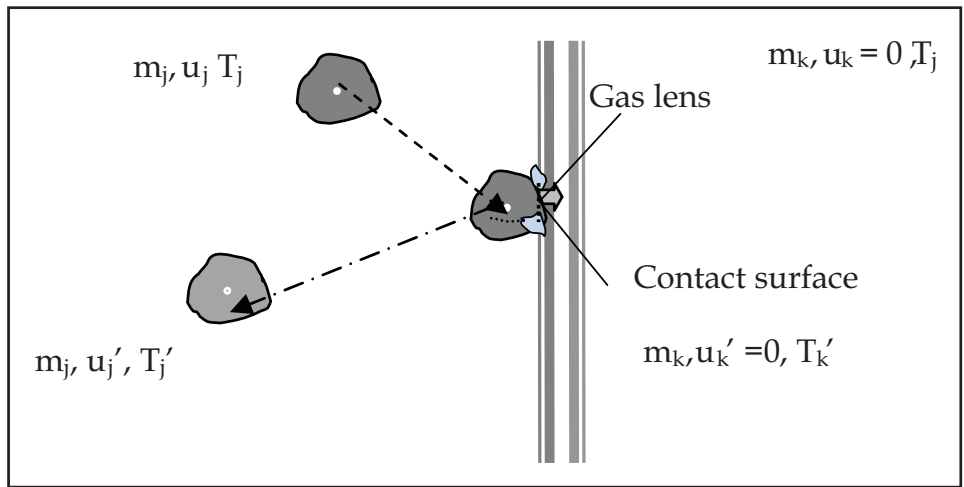

Fig. 2. Collision of an irregular particle with the wall

Now accounting for the probability that a particle is colliding with the surface in the time interval $(t, t+\Delta t)$

$$
\Delta E(\mathbf{x}, t)=\frac{m}{2 N(\mathbf{x}, t)} \int_{0}^{1}\left(e^{2}-1\right) f_{e}(e) d e \iint_{\boldsymbol{U}_{\times} T} u_{j}^{2} \hat{n}\left(\mathbf{x}, \mathbf{u}_{j}, T, t\right) d \mathbf{u}_{j} d T \cdot \Delta t
$$

Making use of the Maxwellian distribution and the hemi-spherical symmetry

$$
d \mathbf{u}_{j}=2 \pi u_{j}^{2} d u_{j}
$$

we obtain

$$
\Delta E(x, t)=\frac{\pi m \int_{0}^{1}\left(e^{2}-1\right) f_{e}(e) d e}{N(x, t)\left(\frac{2}{3} \pi\left\langle u^{2}\right\rangle\right)^{\frac{3}{2}}} \iint_{U \times T} n(x, T, t) u_{j}^{4} \exp \left(-\frac{u_{j}^{2}}{\frac{2}{3}\left\langle u^{2}\right\rangle}\right) d u_{j} d T \cdot \Delta t
$$

that results in the following rate of temperature change for a particle and the wall

$$
\frac{d T}{d t}=\frac{m\left\langle\mathbf{u}^{2}\right\rangle}{8 m_{l} c_{l}} \int_{0}^{1}\left(e^{2}-1\right) f_{e}(e) d e, \quad \imath=p, w
$$

\subsection{Particle-wall heat transfer by collisions}

When a particle and the wall are two colliding bodies, as it shown in Fig. 2, then introducing the notation 


$$
m_{j} c_{j}=m_{p} c_{p}, m_{k} c_{k}=m_{w} c_{w}, p_{j}=p_{p}=\frac{m_{w} c_{w}}{m_{p} c_{p}+m_{w} c_{w}}, p_{k}=p_{w}=\frac{m_{p} c_{p}}{m_{p} c_{p}+m_{w} c_{w}}
$$

as well as $T_{j 0}=T_{p}{ }^{\prime}$ and $T_{k 0}=T_{w}{ }^{\prime}$, we can write

$$
\omega:=\omega_{p w}:=\omega_{w p}:=1-\exp \left[\frac{-h_{p w} a_{p w} \theta_{p w}\left(m_{p} c_{p}+m_{w} c_{w}\right)}{m_{p} c_{p} m_{w} c_{w}}\right]
$$

Now, instead of Eq. (34) we have

$$
T_{p}(\theta)=p_{w} \omega_{p w}\left(T_{w}{ }^{\prime}-T_{p}{ }^{\prime}\right)+T_{p}{ }^{\prime}
$$

from which

$$
\frac{T_{p}-T_{p}{ }^{\prime}}{p_{w} \omega_{p w}}+T_{p}{ }^{\prime}=T_{w}{ }^{\prime}
$$

Eq.(51) expresses the fact that in heat transfer process between a particle and the wall, characterized with parameter $\omega_{p w}$, the temperature $T_{p}{ }^{\prime}$ of the particle becomes $T_{p}$ if the temperature of the wall was $T_{w}{ }^{\prime}$. As a consequence, the conversion distribution function of the particle-wall heat transfer for particles takes the form

$$
B_{p w}\left(T_{p}{ }^{\prime}, T_{p} \mid T_{w}{ }^{\prime}, \omega_{p w}\right)=\mathbf{1}_{T_{p}}\left[\left(\frac{T_{p}-T_{p}{ }^{\prime}}{p_{w} \omega_{p w}}+T_{p}{ }^{\prime}\right)-T_{w}{ }^{\prime}\right]
$$

thus the density function becomes

$$
b_{p w}\left(T_{p}{ }^{\prime}, T_{p} \mid T_{w}{ }^{\prime \prime}, \omega_{p w}\right) d T_{p}=\delta_{T_{p}}\left[\left(\frac{T_{p}-T_{p}{ }^{\prime}}{p_{w} \omega_{p w}}+T_{p}{ }^{\prime}\right)-T_{w}{ }^{\prime}\right] \frac{1}{p_{w} \omega_{p w}} d T_{p}
$$

Finally, in the particle-wall collisional heat transfer we consider the process from the side of the wall. Then Eq.(34) is rewritten as

$$
\frac{T_{w}-T_{w}{ }^{\prime}}{p_{p} \omega_{p w}}+T_{w}{ }^{\prime}=T_{p}{ }^{\prime}
$$

based on which, since the change of the wall temperature is related to $T_{w}(t)$ which is fixed at the moment $t$ the conversion distribution function becomes

$$
B_{w p}\left(T_{w}{ }^{\prime}, T_{w} \mid T_{p}{ }^{\prime}, \omega_{w p}\right)=\mathbf{1}_{T_{w}}\left[\left(\frac{T_{w}-T_{w}{ }^{\prime}}{p_{p} \omega_{w p}}+T_{w}{ }^{\prime}\right)-T_{p}{ }^{\prime}\right]
$$

while the conversion density function 


$$
b_{w p}\left(T_{w}{ }^{\prime}, T_{w} \mid T_{p}{ }^{\prime}, \omega_{w p}\right) d T_{w}=\delta_{T_{w}}\left[\left(\frac{T_{w}-T_{w}{ }^{\prime}}{p_{p} \omega_{w p}}+T_{w}{ }^{\prime}\right)-T_{p}{ }^{\prime}\right] \frac{1}{p_{p} \omega_{w p}} d T_{w}
$$

At this moment all collisional constitutive expressions have been derived and the heat balance model of the process, applying the population balance equation (15) can be determined.

\section{Moment equation model}

Substituting the constitutive equations into the $(3+1) \mathrm{D}$ population balance equation $(15)$ we obtain

$$
\begin{aligned}
& \frac{\partial n(\mathbf{x}, T, t)}{\partial t}+\frac{\partial}{\partial T}\left[\frac{d T}{d t} n(\mathbf{x}, T, t)\right]=\nabla_{\mathbf{x}} \cdot \mathbf{D}_{\mathbf{x}} \nabla_{\mathbf{x}} n(\mathbf{x}, T, t)-\nabla_{\mathbf{x}} \cdot\left[\mathbf{u}_{\mathbf{x}} n(\mathbf{x}, T, t)\right]- \\
& -S_{p p} n(\mathbf{x}, T, t)+\int_{0}^{1} \frac{2 S_{p p}}{\omega_{p p} N(x, t)} \int_{T_{\min }}^{T_{\max }} n\left[\mathbf{x},\left(\frac{2(T-y)}{\omega_{p p}}+y\right), t\right] n(\mathbf{x}, y, t) f_{\omega_{p p}}\left(\omega_{p p}\right) d y d \omega_{p p}
\end{aligned}
$$

where the second term describes the change of population density function due to continuous thermal interactions of particles with gas and energy dissipation generated by particle-particle collisions

$$
\frac{d T}{d t}=\frac{a_{p g} h_{p g}}{m_{p} c_{p}}\left[T_{g}(\mathbf{x}, t)-T\right]+\frac{\left\langle\mathbf{u}^{2}\right\rangle}{8 c_{p}} \int_{0}^{1}\left(e^{2}-1\right) f_{e}(e) d e
$$

Similarly, for the solid surface the boundary condition (16) becomes

$$
\left.d Q_{p w}(\mathbf{x}, t)\right|_{\mathcal{S}}=S_{p w} c_{p} m_{p}\left[-\left.n(\mathbf{x}, T, t)\right|_{\mathcal{S}}+\int_{0}^{1} n\left(\frac{T-p_{w} \omega_{p w} T_{w}(\mathbf{x}, t)}{1-p_{w} \omega_{p w}}, t\right) \frac{f_{\omega_{p w}}\left(\omega_{p w}\right)}{1-p_{w} \omega_{p w}} d \omega_{p w}\right]_{\mathcal{S}} d t
$$

while the boundary condition for surfaces playing the role of input and output of the system takes the form

$$
\left.n(\mathbf{x}, T, t)\right|_{\mathcal{S}}=\mathbf{u}_{\mathbf{x}} n(\mathbf{x}, T, t)-\left.\mathbf{D}_{\mathbf{x}} \cdot \nabla_{\mathbf{x}} n(\mathbf{x}, T, t)\right|_{\mathcal{S}}
$$

where $n(\mathbf{x}, T, t)$ is a given function stimulating the system or specifying the surface conditions.

This is a boundary value problem of the $(3+1) \mathrm{D}$ integral-differential equation (57). This is still a cognitive model and it appears to be too complex for numerical solution. However, accounting for the constitutive expressions of the collision heat transfer terms a useful closed moment equation hierarchy can be determined introducing the moments with respect to the temperature variable

$$
\mu_{k}(\mathbf{x}, t)=\int_{T_{\min }}^{T_{\max }} T^{k} n(\mathbf{x}, T, t) d T, k=0,1,2 \ldots
$$


where $N(\mathbf{x}, t)=\mu_{0}(\mathbf{x}, t)$ and $\langle T\rangle(\mathbf{x}, t)=\mu_{1}(\mathbf{x}, t) / \mu_{0}(\mathbf{x}, t)$ denote, respectively, the total number and the mean temperature of particles in the vicinity of position $x$ at time $t$. In this case the first two moments have physical meaning while by means of the higher order ones the temperature distribution can be specified more exactly.

The infinite set of moment equations becomes

$$
\begin{aligned}
& \frac{\partial \mu_{k}(\mathbf{x}, t)}{\partial t}-\frac{k a_{p g} h_{p g}}{c_{p} m_{p}}\left[\mu_{k-1}(\mathbf{x}, t) T_{g}(\mathbf{x}, t)-\mu_{k}(\mathbf{x}, t)\right]-\frac{k}{c_{p} m_{p}} \Delta H_{c o l l}^{p p} \mu_{k-1}(\mathbf{x}, t)= \\
& =\nabla_{\mathbf{x}} \cdot \mathbf{D}_{\mathbf{x}} \nabla_{\mathbf{x}} \mu_{k}(\mathbf{x}, t)-\nabla_{\mathbf{x}} \cdot\left[\mathbf{u}_{\mathbf{x}} \mu_{k}(\mathbf{x}, t)\right]-S_{p p} \mu_{k}(\mathbf{x}, t)+ \\
& +\frac{S_{p p}}{\mu_{0}(\mathbf{x}, t)} \sum_{j=0}^{k} b_{j k}^{p p} \mu_{j}(\mathbf{x}, t) \mu_{k-j}(\mathbf{x}, t), \quad k=0,1,2, \ldots
\end{aligned}
$$

subject to the initial conditions

$$
\mu_{k}(\mathbf{x}, 0)=\mu_{k, 0}(\mathbf{x}), \quad k=0,1,2, \ldots
$$

In Eqs (62) $\Delta H_{\text {coll }}^{p p}$ denotes the internal heat source generated by particle-particle collisions, in correspondence with Eq.(27), and the coefficients of the collision terms are

$$
b_{j k}^{p p}=\int_{0}^{1}\left(\begin{array}{l}
k \\
j
\end{array}\right)\left(\frac{\omega_{p p}}{2}\right)^{j}\left(1-\frac{\omega_{p p}}{2}\right)^{k-j} f_{\omega_{p p}}\left(\omega_{p p}\right) d \omega_{p p}, k=0,1,2, \ldots, j=1,2, \ldots k
$$

while the boundary conditions become

$$
\left.d \mu_{k}(\mathbf{x}, t)\right|_{\mathcal{S}}=\left.S_{p w} \sum_{j=0}^{k} b_{j k}^{p w} \mu_{j}(\mathbf{x}, t) T_{w}^{k-j}(\mathbf{x}, t)\right|_{\mathcal{S}} \cdot d t, \quad k=0,1,2, \ldots
$$

and

$$
\left.\mu_{k}(\mathbf{x}, t)\right|_{s}=\mathbf{u}_{\mathbf{x}} \mu_{k}(\mathbf{x}, t)-\left.\mathbf{D}_{\mathbf{x}} \cdot \nabla_{\mathbf{x}} \mu_{k}(\mathbf{x}, t)\right|_{S}
$$

where

$$
b_{j k}^{p w}=\int_{0}^{1}\left(\begin{array}{l}
k \\
j
\end{array}\right)\left(1-p_{w} \omega_{p w}\right)^{j}\left(p_{w} \omega_{p w}\right)^{k-j} f_{\omega_{p w}}\left(\omega_{p w}\right) d \omega_{p w}, \quad k=0,1,2, \ldots, j=1,2, \ldots k
$$

Here $\mu_{k}(\mathbf{x}, t)$ denotes the $k^{\text {th }}$ order moment of a given function stimulating the system across the inlet surface and that providing the outlet surface conditions.

Specifying the appropriate symmetry conditions for the spatial terms in Eq.(62) results in 3D, 2D and 1D applicative, i.e. purpose-oriented moment equation models for different gasparticle processing systems.

When applying the moment equation model, the computational results are assessed by means of the first three of the normalized moments

$$
m_{k}(\mathbf{x}, t)=\frac{\mu_{k}(\mathbf{x}, t)}{\mu_{0}(\mathbf{x}, t)}, k=0,1,2 .
$$


Here, beside the zero order and the normalized first order moments, i.e. the total number and mean temperature of particles, the variance defined as

$$
\sigma^{2}(\mathbf{x}, t)=\frac{\mu_{2}(\mathbf{x}, t)}{\mu_{0}(\mathbf{x}, t)}-\left(\frac{\mu_{1}(\mathbf{x}, t)}{\mu_{0}(\mathbf{x}, t)}\right)^{2}=m_{2}(\mathbf{x}, t)-m_{1}^{2}(\mathbf{x}, t)
$$

is used for characterizing the temperature distribution of particles.

\section{Heat transfer in bubbling fluidized beds}

Gas-solid fluidized beds may operate in several different flow regimes: bubbling, slug flow, turbulent and fast fluidization. With increasing superficial velocity there is a transition between the lower velocity bubbling and higher velocity turbulent fluidization states (Thompson et al., 1999, Bi et al., 2000). In the bubbling regime, as the gas flow rate increases the total volume of bubbles also increases and accounting for their coalescence and breakage, the bubble phase can be described by an axial dispersion model containing no particles. The bubbling regime, however, is characterized by intensive pressure fluctuations so that the dense emulsion phase, containing the particle population and being in intensive motion can be modeled as a perfectly stirred part of the bed as it is shown schematically in Fig. 3. At higher velocities, because of disappearance of bubbles the amplitudes of pressure fluctuations decrease significantly, and the distribution of particles along the height of the vessel becomes smoother. In this case the spatial distribution of particles is described by an axial dispersion model too, as it was analyzed by Süle et al. (2010).

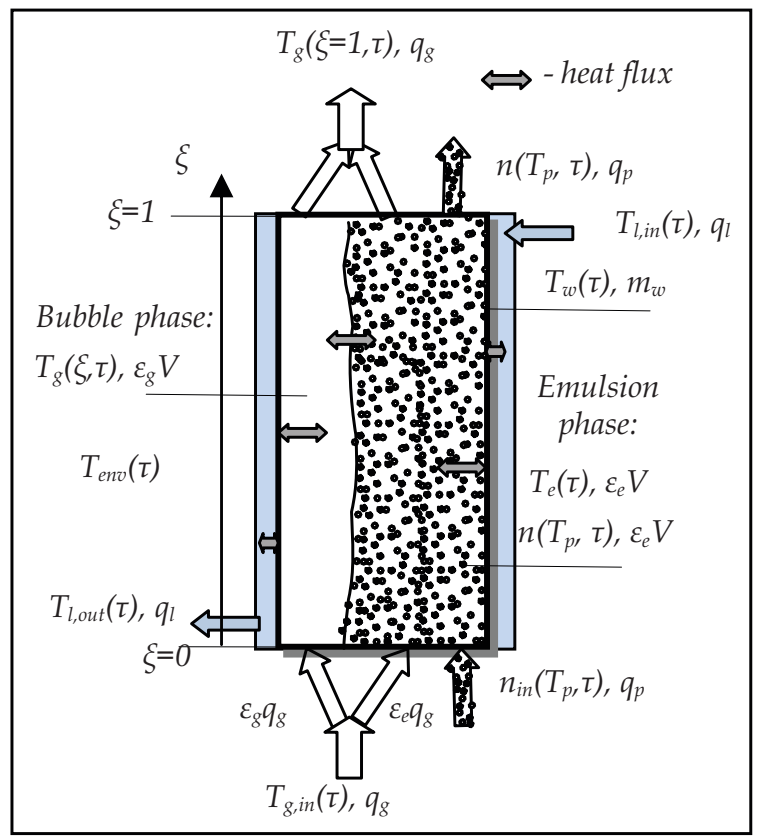

Fig. 3. Schematics of a bubbling fluidized bed 
As regards the model of bubbling fluidization we start with the following assumptions.

- The bubble phase is described by an axial dispersion model, and is connected thermally with the emulsion phase through the gas and with the wall.

- The emulsion phase is a perfectly stirred cell containing the homogeneously distributed particle population. Inside the emulsion phase heat transfer occurs between the gas and particles, and there exists thermal connection of the gas and particles of the emulsion phase and the wall.

- Homogeneous temperature distribution is assumed in the wall through which the bed is connected thermally with the jacket filled with liquid.

- Liquid through the jacket flows with constant flow rate and is assumed to be thermally homogeneous.

- The bubbling fluidized bed is operated continuously.

Introducing the dimensionless variables and parameters

$$
\xi=\frac{x}{X}, \bar{t}_{p}=\frac{\varepsilon_{e} V}{q_{p}}, \tau=\frac{t}{\bar{t}_{p}}, P e_{b}=\frac{u_{b} X}{D_{b}}, \bar{t}_{b}=\frac{X}{u_{b}}
$$

the axial dispersion model for the bubble phase is written as

$$
\begin{aligned}
& \frac{\partial T_{b}(\xi, \tau)}{\partial \tau}=\frac{\bar{t}_{p}}{\bar{t}_{b}} \frac{\partial}{\partial \xi}\left(\frac{1}{P e_{b}} \frac{\partial T_{b}(\xi, \tau)}{\partial \xi}-T_{b}(\xi, \tau)\right)-K_{b e} \bar{t}_{p} \int_{0}^{1}\left(T_{b}(\xi, \tau)-T_{e}(\tau)\right) d \xi \\
& -K_{b w} \bar{t}_{p} \int_{0}^{1}\left(T_{b}(\xi, \tau)-T_{w}(\xi, \tau)\right), \quad \tau>0, \xi \in(0,1)
\end{aligned}
$$

while the corresponding boundary conditions take the forms

$$
\frac{u_{b, i n}}{u_{b}} T_{b, i n}(\tau)=T_{b}(0+, \tau)-\frac{1}{P e_{b}} \frac{\partial T_{b}(0+, \tau)}{\partial \xi}, \frac{\partial T_{b}(1-, \tau)}{\partial \xi}=0
$$

In Eq. (70)

$$
K_{b e}=\frac{a_{b e} h_{b e}}{\rho_{g} c_{g}} \text { and } K_{b w}=\frac{a_{b w} h_{b w}}{\rho_{g} c_{g}}
$$

denote the coefficients of heat transfers between the bubbles and, respectively, the emulsion phase and the wall.

Integrating both sides of Eq. (57) over the volume of the emulsion phase $\varepsilon_{e} V$ and taking into consideration the boundary conditions we obtain for the population of particles of the emulsion phase

$$
\begin{aligned}
& \frac{\partial n(T, t)}{\partial t}+\frac{\partial}{\partial T}\left[\frac{d T}{d t} n(T, t)\right]=\frac{q_{p}}{\varepsilon_{e} V}\left[n_{i n}(T, t)-n(T, t)\right]-\left(S_{p p}+S_{p w}\right) n(T, t) \\
& +\frac{2}{N(t)} \int_{0}^{1} \frac{S_{p p}}{\omega_{p p}} \int_{T_{\min }}^{T_{\max }} n\left[\left(\frac{2(T-y)}{\omega_{p p}}+y\right), t\right] n(y, t) f_{\omega_{p p}}\left(\omega_{p p}\right) d y d \omega_{p p}+ \\
& +\int_{0}^{1} \frac{S_{p w}}{1-p_{w} \omega_{p w}} \int_{T_{\min }}^{T_{\max }} n\left(\frac{y-p_{w} \omega_{p w} T_{w}(t)}{1-p_{w} \omega_{p w}}, t\right) f_{\omega_{p w}}\left(\omega_{p w}\right) d y d \omega_{p w}
\end{aligned}
$$


where

$$
n(T, t)=\frac{1}{\varepsilon_{e} V} \int_{\varepsilon_{e} V} n(x, T, t) d V
$$

and

$$
\frac{d T}{d t}=\frac{a_{p e} h_{p e}}{m_{p} c_{p}}\left[T_{e}(t)-T\right]+\frac{\Delta H}{m_{p} c_{p}}
$$

where $\Delta H=\Delta H_{\text {coll }}^{p p}+\Delta H_{\text {coll }}^{p w}$ is the overall heat effects generated by the particle-particle and particle-wall collisions, in correspondence with Eqs. (27) and (47). Naturally, when the particles are homogeneously distributed in the emulsion phase then $n(T, t) \equiv n(\mathbf{x}, T, t)$.

In this case the moment equations (62), taking into account Eq. (72) and written using the dimensionless variables and parameters are as follows.

Zero order moment:

$$
\frac{d \mu_{0}(\tau)}{d \tau}=\mu_{0, i n}(\tau)-\mu_{0}(\tau)
$$

First order moment:

$$
\begin{aligned}
& \frac{d \mu_{1}(\tau)}{d \tau}=\mu_{1, i n}(\tau)-\mu_{1}(\tau)+K_{p e} \bar{t}_{p}\left(\mu_{0}(\tau) T_{e}(\tau)-\mu_{1}(\tau)\right)+ \\
& +S_{p w} p_{w} m_{1, \omega_{p w}} \bar{t}_{p}\left(\mu_{1}(\tau)-T_{w}(\tau) \mu_{0}(\tau)\right)+\frac{\bar{t}_{p}}{m_{p} c_{p}} \Delta H \mu_{0}(\tau)
\end{aligned}
$$

Second order moment:

$$
\begin{aligned}
\frac{d \mu_{2}(\tau)}{d \tau}= & \mu_{2, i n}(\tau)-\mu_{2}(\tau)+2 K_{p e} \bar{t}_{p}\left(\mu_{1}(\tau) T_{e}(\tau)-\mu_{2}(\tau)\right)+\frac{2 \bar{t}_{p}}{m_{p} c_{p}} \Delta H \mu_{1}(\tau)+ \\
& +S_{p p} \kappa_{p p} \bar{t}_{p}\left(\frac{\mu_{1}^{2}(\tau)}{\mu_{0}(\tau)}-\mu_{2}(\tau)\right)+2 S_{p w} p_{w} m_{1, \omega_{p w}} \bar{t}_{p}\left[T_{w}(\tau) \mu_{1}(\tau)-\mu_{2}(\tau)\right]+ \\
& +S_{p w} p_{w}^{2} m_{2, \omega_{p w}} \bar{t}_{p}\left(\mu_{2}(\tau)-2 T_{w}(\tau) \mu_{1}(\tau)+T_{w}^{2}(\tau) \mu_{0}(\tau)\right)
\end{aligned}
$$

where the coefficients of terms representing the collision particle-particle and particle-wall heat transfers are expressed by means of expectations and variances of random parameters $\omega_{p p}$ and $\omega_{p w}$. In Eqs. (75) and (76), we have

$$
K_{p e}=\frac{a_{p e} h_{p e}}{m_{p} c_{p}} \text { and } \kappa_{p p}=m_{1, \omega_{p p}}\left(1-\frac{m_{1, \omega_{p p}}}{2}\right)-\frac{\sigma_{\omega_{p p}}^{2}}{2}
$$

The differential equation for the gas contents in the emulsion phase is given as 


$$
\begin{aligned}
& \frac{d T_{e}(\tau)}{d \tau}=\frac{\bar{t}_{p}}{\bar{t}_{e}}\left(T_{e, i n}(\tau)-T_{e}(\tau)\right)-K_{w e} \bar{t}_{p}\left(T_{e}(\tau)-T_{w}(\tau)\right)-K_{p e} \bar{t}_{p}\left(\mu_{0}(t) T_{e}(\tau)-\mu_{1}(\tau)\right) \\
& +K_{b e} \bar{t}_{p} \int_{0}^{1}\left(T_{b}(\xi, \tau)-T_{e}(\tau)\right) d \xi
\end{aligned}
$$

where

$$
K_{w e}=\frac{h_{w e} a_{w e}}{\rho_{g} c_{g}}, K_{p e}=\frac{h_{p e} a_{p e}}{\rho_{g} c_{g}}, K_{b e}=\frac{h_{b e} a_{b e}}{\rho_{g} c_{g}}
$$

Finally the balance equation for the wall takes the form:

$$
\begin{aligned}
& \frac{d T_{w}(\tau)}{d \tau}=K_{w b} \bar{t}_{p} \int_{0}^{1}\left(T_{b}(\xi, \tau)-T_{w}(\tau)\right)+K_{p w} \bar{t}_{p}\left(\mu_{1}(\tau)-T_{w}(t) \mu_{0}(\tau)\right)+ \\
& +K_{w e} \bar{t}_{p}\left(T_{e}(\tau)-T_{w}(\tau)\right)-K_{w l} \bar{t}_{p}\left(T_{w}(\tau)-T_{l}(\tau)\right)
\end{aligned}
$$

where

$$
K_{w b}=\frac{a_{w b} h_{w b}}{\rho_{w} c_{w}}, \quad K_{p w}=\frac{S_{p w} p_{p} m_{1, \omega_{p w}}}{\rho_{w} c_{w}}, K_{w e}=\frac{a_{w e} h_{w e}}{\rho_{w} c_{w}}, K_{w, e n v}=\frac{a_{w, e n v} h_{w, e n v}}{\rho_{w} c_{w}}
$$

while for the liquid we have:

$$
\frac{d T_{l}(\tau)}{d \tau}=\frac{\bar{t}_{p}}{\bar{t}_{l}}\left(T_{l, i n}(\tau)-T_{l}(\tau)\right)-K_{w l} \bar{t}_{p}\left(T_{l}(\tau)-T_{w}(\tau)\right)
$$

where

$$
K_{w l}=\frac{a_{w l} h_{w l}}{\rho_{l} c_{l}}
$$

\section{Simulation results and discussion}

Numerical experiments for studying the behavior of bubbling fluidized bed were carried out in MATLAB environment. The basic process parameters and hydrodynamic properties of the fluidized bed were computed using the values and correlations given by Lakatos et. al. (2008) while the heat source generated by the energy loss due to collisions was taken constant. The details of numerical experimentation will be presented elsewhere.

Fig. 4. presents the time evolution and steady state values of the variance of temperature of particles, defined by Eq. (69), depending on the interparticle collision frequency $S_{p p}$. Initially the temperature of particle population was homogeneous but the particle temperature input was generated as a step function having two different temperatures $T_{p, i n}^{(1)}=20^{\circ} \mathrm{C}$ and $T_{p, i n}^{(2)}=160^{\circ} \mathrm{C}$, respectively, which were totally segregated from each other. Such temperature distribution is described by the input population density function having the form 


$$
n_{\text {in }}(T, \tau)=\phi M_{0, \text { in }} \delta\left(T-T_{\text {in }}^{(1)}\right)+(1-\phi) M_{0, \text { in }} \delta\left(T-T_{\text {in }}^{(2)}\right), \quad 0 \leq \varphi \leq 1
$$

where $M_{0, i n}=10^{8} / \mathrm{m}^{3}$ is the total number of particles in a unit volume, and $\varphi=0.6$ is the ratio of particles having different temperatures.

Fig. 4 shows that there may remain rather significant inhomogeneities in the temperature distribution of particles in the bed. These inhomogeneities are decreasing with increasing interparticle collision frequency therefore the particle-particle interaction play important role in homogenization of temperature inside the population of particles.

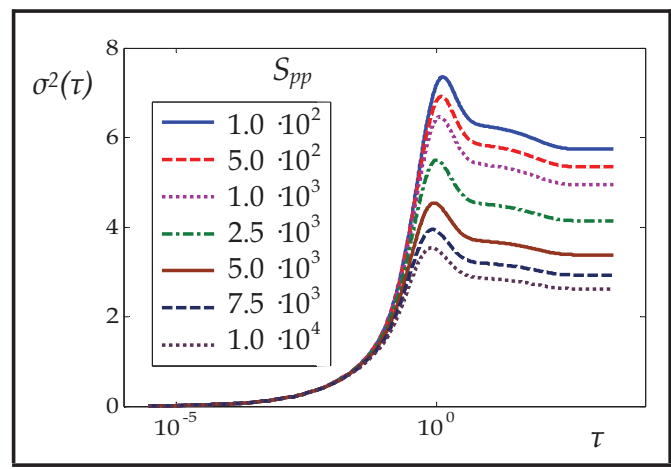

Fig. 4. Time evolution of the variance of temperature distribution of the particle population

Fig. 5 presents the time evolution of the temperatures of characteristic parts of the bubbling bed, i.e. mean temperature of particles, the temperatures of the bubble phase and the gas in the emulsion phase, as well as the temperatures of the wall and liquid in the jacket. In this case the input temperature of gas was $180{ }^{\circ} \mathrm{C}$ and the initial temperature of gas in the emulsion and bubble phases were of the same values. It is seen how the temperature profiles vary in interconnections of the different parts of the bed. In steady state, under the given heat transfer resistance conditions of the particle population, the wall and liquid is heated practically only by the gas in the emulsion phase while the bubbles flow through the bed without losing heat assuring only the well stirred state of the emulsion phase.

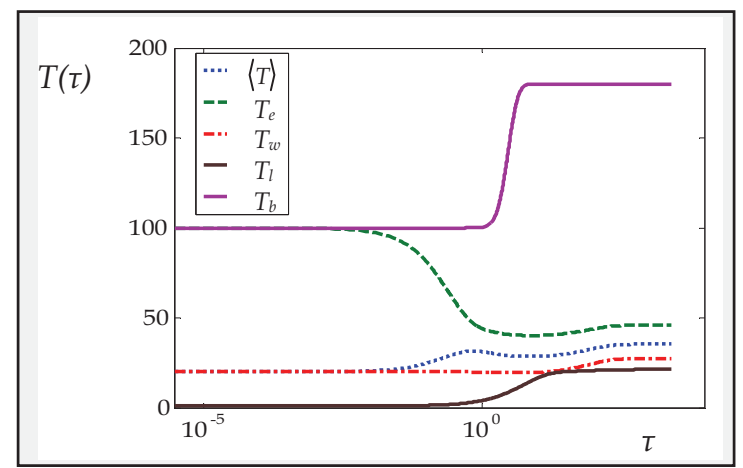

Fig. 5. Time evolution of the temperatures of characteristic parts of the bubbling bed in interrelations with each other 
In Fig. 5, bubble temperature transient is shown only for the bed output indicating here a rather sharp front. Naturally, the temperature front of bubbles evolves progressively along the bed as it shown in Fig. 6 at different axial coordinates showing that in transient states the bubble phase also plays role in the heating process.

However the bubble evolves transient states

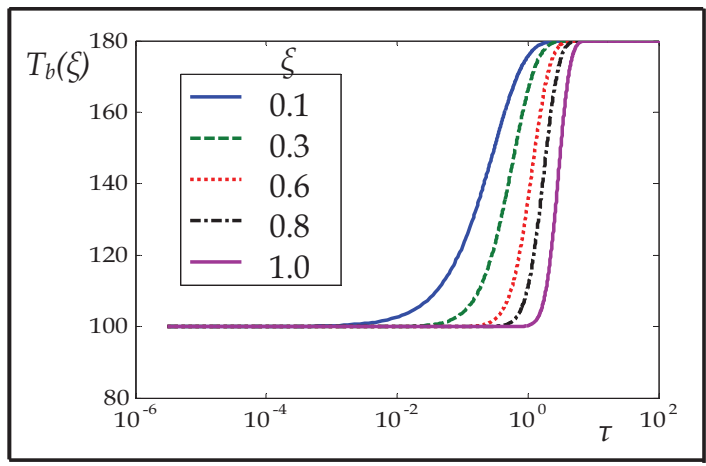

Fig. 6. Progression of evolution of the temperature front of the bubble phase along the axial coordinate of the bed

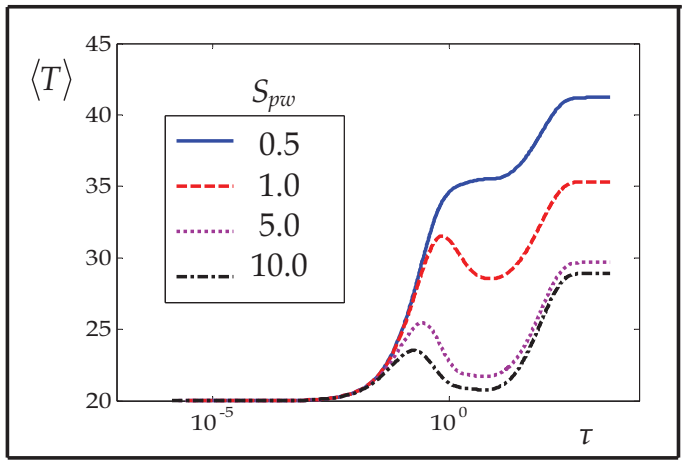

Fig. 7. Time evolution of the mean temperature of particle population as a function of the particle-wall collision frequency

Eq. (76) shows clearly that when the number of particles is constant, i.e. under steady state hydrodynamic conditions interparticle heat transfer does not influence the mean value of temperature of the particle population but, as it is demonstrated by Fig. 4, it affects the variance. However, as Fig. 7 gives an evidence of that the mean value of temperature of the particle population depends on the particle-wall collision frequency. This figure indicates also that because of the heat transfer interrelations of different parts of the bed oscillations may arise in the transient processes which becomes smoothed as the particle-wall collision frequency decreases. At the same time, in this case the particle-wall collision frequency affects also the variance of temperature distribution of the particle population as it is shown in Fig. 8. It is seen that with increasing particle-wall collision frequency the variance de- 
creases. i.e. increasing particle-wall heat transfer intensity gives rise to smaller inhomogeneites in the temperature distribution of particles.

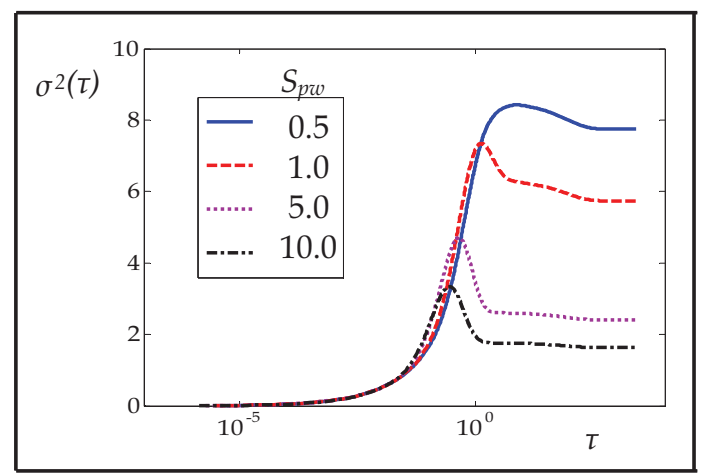

Fig. 8. Time evolution of the variance of temperature distribution of the particle population as a function of the particle-wall collision frequency

\section{Conclusion}

The spatially distributed population balance model presented in this chapter provides a tool of modeling heat transfer processes in gas-solid processing systems with interparticle and particle-wall interactions by collisions. Beside the gas-solid, gas-wall and wall-environment heat transfers the thermal effects of collisions have also been included into the model. The basic element of the model is the population density function of particle population the motion of which in the space of position and temperature variables is governed by the population balance equation.

The population density function provides an important and useful characterization of the temperature distribution of particles by means of which temperature inhomogeneities and developing of possible hot spots can be predicted in particulate processes. In generalized form the model can serve for cognitive purposes but by specifying appropriate symmetry conditions useful applicative, i.e. purpose-oriented models can be obtained.

The second order moment equation model, obtained from the infinite hierarchy of moment equations generated by the population balance equation, as an applicative model can be applied successfully for analyzing the thermal properties of gas-solid processing systems by simulation. The first two moments are required to formulate the heat balances of the particulate system while the higher order moments are of use for characterizing the process in more detail.

Applicability of the second order moment equation model was demonstrated by modeling and studying the behavior of bubbling fluidization by numerical experiments. It has proved that collision particle-particle and particle-wall heat transfers contribute to homogenization of the temperature of particle population to a large extent. The particle-particle heat transfer no affects the mean temperature of particle population and, in fact, no influences any of temperatures of the system whilst the particle-wall heat transfer collisions exhibits significant influence not only on the steady state temperatures but on the transient processes of the system as well. It has been demonstrated that the second order moment equation 
model can be effectively used to analyze both the dynamical and steady state processes of bubbling fluidization

\section{Acknowledgement}

This work was supported by the Hungarian Scientific Research Fund under Grant K 77955.

\section{Symbols}

$\begin{array}{lll}a & - & \text { surface area, } \mathrm{m}^{2} \\ c & - & \text { specific heat, } \mathrm{J} \mathrm{kg}^{-1} \mathrm{~K}^{-1} \\ D & - & \text { dispersion coefficient, } \mathrm{m}^{2} \mathrm{~s}^{-1} \text {, bed diameter, } \mathrm{m} \\ e & - & \text { coefficient of restitution } \\ f & - & \text { probability density function } \\ h & - & \text { enthalpy, } \mathrm{J} ; \text { heat transfer coefficient } \mathrm{W} \mathrm{m}^{-2} \mathrm{~K}^{-1} \\ K & - & \text { aggregate rate coefficient of heat transfer } \\ k & - & \text { thermal conductivity, } \mathrm{W} \mathrm{m} \mathrm{m}^{-1} \mathrm{~K}^{-1} \\ m & - & \text { mass, kg } \\ m_{k} & - & \text { normalized } k^{\text {th }} \text { order moment of particle temperature } \\ N & - & \text { number of particles, no } \mathrm{m}^{-3} \\ n & - & \text { population density function, no } \mathrm{m}^{-3} \mathrm{~K}-1 \\ p_{p} & - & \text { weight parameter } \\ p_{w} & - & \text { weight parameter } \\ P e & - & \text { Peclet number } \\ q & - & \text { volumetric flow rate, } \mathrm{m}^{3} \mathrm{~s}^{-1} \\ S & - & \text { frequency of collisions, } \mathrm{s}^{-1} \\ T & - & \text { temperature, } \mathrm{K} \\ t & - & \text { time, } \mathrm{s} \\ u & - & \text { linear velocity, } \mathrm{m} \mathrm{s}-1 \\ v & - & \text { volume, m } \\ V & - & \text { volume, m } \\ x & - & \text { axial coordinate, } \mathrm{m} \\ \bar{t} & & \text { mean residence time, } \mathrm{s} \\ \langle.\rangle & & \text { mean value } \\ \mathbf{1} & - & \text { Heaviside function }\end{array}$

Greek symbols

$\begin{array}{lll}\beta & - & \text { aggregate heat transfer coefficient } \\ \rho & - & \text { density, } \mathrm{kg} \mathrm{m}^{-3} \\ \delta & - & \text { Dirac delta function } \\ \kappa & - & \text { parameter } \\ \omega & - & \text { random variable characterizing collision heat transfer } \\ \mu_{k} & - & k^{\text {th }} \text { order moment of particle temperature } \\ \varepsilon & - & \text { volumetric fraction } \\ \xi & - & \text { dimensionless axial coordinate } \\ \tau & - & \text { dimensionless time }\end{array}$




$\begin{array}{lll}\sigma^{2} & - & \text { variance of the temperature of particle pop } \\ \text { Subscripts and } & \text { superscripts } \\ c & - & \text { critical value } \\ e & - & \text { emulsion phase; coefficient of restitution } \\ g & - & \text { gas } \\ \text { in } & - & \text { input } \\ b & - & \text { bubble } \\ \max & - & \text { maximal value } \\ \text { min } & - & \text { minimal value } \\ p & - & \text { particle } \\ p g & - & \text { particle-gas } \\ p p & - & \text { particle-particle } \\ p w & - & \text { particle-wall } \\ w & - & \text { wall } \\ w b & - & \text { wall-gas }\end{array}$

\section{References}

Bi, H.T., Ellis, N., Abba, A. \& Grace, J.R. (2000). A state-of-the-art review of gas-solid turbulent fluidization. Chem. Eng. Sci., 55, 4789-4825.

Boulet, P, Moissette, S., Andreaux, R. \& Osterlé, B., (2000). Test of an Eulerian-Lagrangian simulation of wall heat transfer in a gas-solid pipe flow. Int. J. Heat Fluid Flow, 21, 381-387.

Chagras, V., Osterlé, B. \& Boulet, P. (2005). On heat transfer in gas-solid pipe flows: Effects of collision induced alterations on the flow dynamics. Int. J. Heat Mass Transfer, 48, 1649-1661.

Chang, J. \& Yang, S. (2010). A particle-to-particle heat transfer model dense gas-solid fluidi-zed bed of binary mixtures. Chem. Eng. Res. Des., doi:10.1018/j.cherd.2010.08.004

Delvosalle, C. \& Vanderschuren, J., (1985). Gas-to-particle and particle-to-particle heat transfer in fluidized beds of large particles. Chem. Eng. Sci., 40, 769-779.

Gardiner, C.W.,(1983). Handbook of Stochastic Methods. Springer-Verlag, Berlin.

Gidaspow, D. (1994). Multiphase Flow and Fluidization. Boston: Academic Press.

Lakatos, B.G., Mihálykó, Cs. \& Blickle, T. (2006). Modelling of interactive populations of disperse system. Chem. Eng. Sci., 61, 54-62.

Lakatos, B.G., Süle, Z. \& Mihálykó, Cs. (2008). Population balance model of heat transfer in gas-solid particulate systems. Int. J. Heat Mass Transfer, 51, 1633-1645.

Mansoori, Z., Saffar-Avval, M., Basirat-Tabrizi, H., Ahmadi, G. \& Lain, S. (2002). Thermomechanical modeling of turbulent heat transfer in gas-solid flows including particle col-lisions, Int. J. Heat Fluid Flow Transfer, 23, 792-806.

Mansoori, Z., Saffar-Avval, M., Basirat-Tabrizi, H., Dabir, B. \& Ahmadi, G. (2005). Interparticle heat transfer in a riser of gas-solid turbulent flows. Powder Technol., 159, 35-45.

Martin, H., (1984). Heat transfer between gas fluidized bed of solid particles and the surfaces of immersed heat transfer exchanger elements. Chem. Eng. Proc. 18, 199-223. 
Mihálykó, Cs., Lakatos, B.G., Matejdesz, A. \& Blickle, T., (2004). Population balance model for particle-to-particle heat transfer in gas-solid systems. Int. J. Heat Mass Transfer, $47,1325-1334$.

Molerus, O., (1997). Heat transfer in moving beds with a stagnant interstitial gas. Int. J. Heat Mass Transfer, 40, 4151-4159.

Ramkrishna, D. (2000). Population Balances. Theory and Applications to Particulate Systems in Engineering. San Diego: Academic Press.

Schlünder, E.U., (1984). Heat transfer to packed and stirred beds from the surface of immersed bodies. Chem. Eng. Proc. 18, 31-53.

Sobczyk, K., (1991). Stochastic Differential Equations with Applications to Physics and Engeneering. Kluwer Academic, Amsterdam.

Süle, Z., Lakatos, B.G. \& Mihálykó, Cs., (2009). Axial dispersion/population balance model of heat transfer in turbulent fluidization. in: Jeżowski J., and Thullie, J., (Eds) Proc. 19th ESCAPE. Comp. Aided Chem. Eng. 26, Elsevier, Amsterdam:, 815-820.

Süle, Z., Lakatos, B.G. \& Mihálykó, Cs., (2010). Axial dispersion/population balance model of heat transfer in turbulent fluidization. Comp. Chem. Eng., 34, 753-762.

Süle, Z., Mihálykó, Cs. \& Lakatos, B.G. (2006). Modelling of heat transfer processes in particulate systems. in: W. Marquardt, C. Pantelides (Eds), Proc. 16th ESCAPE and 9th ISPSE. Comp-Aided Chem. Eng. 21A, Elsevier, Amsterdam, 589-594.

Süle, Z., Mihálykó, Cs. \& Lakatos, G.B., (2008). Population balance model of gas-solid fluidized bed heat exchangers. Chem. Proc. Eng., 29, 201-213.

Sun, J. \& Chen, M.M., (1988). A theoretical analysis of heat transfer due to particle impact. Int. J. Heat Mass Transfer, 31, 969-975.

Thompson, M.L., Bi, H. \& Grace, J.R. (1999). A generalized bubbling/turbulent fluidizedbed reactor model. Chem. Eng. Sci., 54, 2175-2185.

Vanderschuren, J. \& Delvosalle, C., (1980). Particle-to-particle heat transfer in fluidized bed drying. Chem. Eng. Sci., 35, 1741-1748 


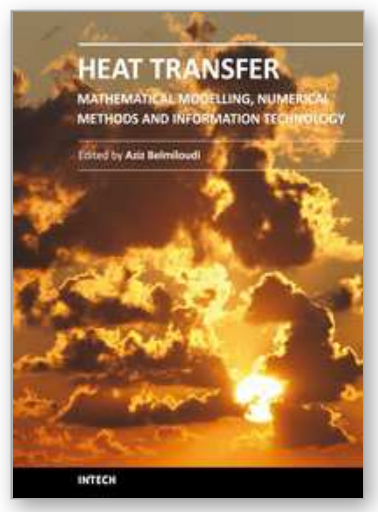

\section{Heat Transfer - Mathematical Modelling, Numerical Methods and Information Technology}

Edited by Prof. Aziz Belmiloudi

ISBN 978-953-307-550-1

Hard cover, 642 pages

Publisher InTech

Published online 14, February, 2011

Published in print edition February, 2011

Over the past few decades there has been a prolific increase in research and development in area of heat transfer, heat exchangers and their associated technologies. This book is a collection of current research in the above mentioned areas and describes modelling, numerical methods, simulation and information technology with modern ideas and methods to analyse and enhance heat transfer for single and multiphase systems. The topics considered include various basic concepts of heat transfer, the fundamental modes of heat transfer (namely conduction, convection and radiation), thermophysical properties, computational methodologies, control, stabilization and optimization problems, condensation, boiling and freezing, with many real-world problems and important modern applications. The book is divided in four sections : "Inverse, Stabilization and Optimization Problems", "Numerical Methods and Calculations", "Heat Transfer in Mini/Micro Systems", "Energy Transfer and Solid Materials", and each section discusses various issues, methods and applications in accordance with the subjects. The combination of fundamental approach with many important practical applications of current interest will make this book of interest to researchers, scientists, engineers and graduate students in many disciplines, who make use of mathematical modelling, inverse problems, implementation of recently developed numerical methods in this multidisciplinary field as well as to experimental and theoretical researchers in the field of heat and mass transfer.

\section{How to reference}

In order to correctly reference this scholarly work, feel free to copy and paste the following:

Béla G. Lakatos (2011). Population Balance Model of Heat Transfer in Gas-Solid Processing Systems, Heat Transfer - Mathematical Modelling, Numerical Methods and Information Technology, Prof. Aziz Belmiloudi (Ed.), ISBN: 978-953-307-550-1, InTech, Available from: http://www.intechopen.com/books/heat-transfermathematical-modelling-numerical-methods-and-information-technology/population-balance-model-of-heattransfer-in-gas-solid-processing-systems

\section{INTECH}

open science | open minds

\section{InTech Europe}

University Campus STeP Ri

Slavka Krautzeka 83/A

51000 Rijeka, Croatia

Phone: +385 (51) 770447

\section{InTech China}

Unit 405, Office Block, Hotel Equatorial Shanghai

No.65, Yan An Road (West), Shanghai, 200040, China 中国上海市延安西路65号上海国际贵都大饭店办公楼 405 单元

Phone: +86-21-62489820 
Fax: +385 (51) 686166

Fax: +86-21-62489821

www.intechopen.com 
(C) 2011 The Author(s). Licensee IntechOpen. This chapter is distributed under the terms of the Creative Commons Attribution-NonCommercialShareAlike-3.0 License, which permits use, distribution and reproduction for non-commercial purposes, provided the original is properly cited and derivative works building on this content are distributed under the same license. 